PROCEEDINGS OF THE

AMERICAN MATHEMATICAL SOCIETY

Volume 126, Number 11, November 1998, Pages 3137-3143

S $0002-9939(98) 04680-2$

\title{
HYERS-ULAM-RASSIAS STABILITY OF JENSEN'S EQUATION AND ITS APPLICATION
}

\author{
SOON-MO JUNG
}

(Communicated by Palle E. T. Jorgensen)

\begin{abstract}
The Hyers-Ulam-Rassias stability for the Jensen functional equation is investigated, and the result is applied to the study of an asymptotic behavior of the additive mappings; more precisely, the following asymptotic property shall be proved: Let $X$ and $Y$ be a real normed space and a real Banach space, respectively. A mapping $f: X \rightarrow Y$ satisfying $f(0)=0$ is additive if and only if $\|2 f[(x+y) / 2]-f(x)-f(y)\| \rightarrow 0$ as $\|x\|+\|y\| \rightarrow \infty$.
\end{abstract}

\section{INTRODUCTION}

In 1940, S. M. Ulam [9] gave a wide ranging talk before the Mathematics Club of the University of Wisconsin in which he discussed a number of important unsolved problems. Among those was the following question concerning the stability of homomorphisms:

Let $G_{1}$ be a group and let $G_{2}$ be a metric group with the metric $d(\cdot, \cdot)$. Given $\varepsilon>0$, does there exist a $\delta>0$ such that if a mapping $h: G_{1} \rightarrow G_{2}$ satisfies the inequality $d(h(x y), h(x) h(y))<\delta$ for all $x, y \in G_{1}$ then a homomorphism $H: G_{1} \rightarrow G_{2}$ exists with $d(h(x), H(x))<\varepsilon$ for all $x \in G_{1}$ ?

The case of approximately additive mappings was solved by D. H. Hyers [2] under the assumption that $G_{1}$ and $G_{2}$ are Banach spaces. In 1978, Th. M. Rassias [6] generalized the result of Hyers as follows:

Let $f: X \rightarrow Y$ be a mapping between Banach spaces and let $0 \leq p<1$ be fixed. If $f$ satisfies the inequality

$$
\|f(x+y)-f(x)-f(y)\| \leq \theta\left(\|x\|^{p}+\|y\|^{p}\right)
$$

for some $\theta \geq 0$ and for all $x, y \in X$, then there exists a unique additive mapping $A: X \rightarrow Y$ such that

$$
\|f(x)-A(x)\| \leq \frac{2 \theta}{2-2^{p}}\|x\|^{p}
$$

for all $x \in X$. If, in addition, $f(t x)$ is continuous in $t$ for each fixed $x \in X$, then $A$ is linear.

Received by the editors March 19, 1997.

1991 Mathematics Subject Classification. Primary 39B72.

Key words and phrases. Hyers-Ulam-Rassias stability, Jensen functional equation.

(C)1998 American Mathematical Society 
Taking this fact into account, the additive functional equation $f(x+y)=f(x)+$ $f(y)$ is said to have the Hyers-Ulam-Rassias stability on $(X, Y)$. This terminology is also applied to the case of other functional equations. For more detailed definitions of such terminology one can refer to [1] and [3].

Throughout this paper, let $X$ and $Y$ be a real normed space and a real Banach space, respectively. According to Theorem 6 in [5], a mapping $f: X \rightarrow Y$ satisfying $f(0)=0$ is a solution of the Jensen functional equation

$$
2 f\left(\frac{x+y}{2}\right)=f(x)+f(y)
$$

if and only if it satisfies the additive Cauchy equation $f(x+y)=f(x)+f(y)$. Hence, the most general continuous solution of the Jensen's equation in $\mathbb{R}$ is $f(x)=a x+b$, where $a$ and $b$ are arbitrary constants.

The first result on the stability of Jensen's equation was obtained by Z. Kominek (see [4]). In fact, he proved the following theorem:

Theorem. Let $D$ be a subset of $\mathbb{R}^{n}$ with non-empty interior. Assume that there exists an $x_{0}$ in the interior of $D$ such that $D_{0}=D-x_{0}$ satisfies the condition $(1 / 2) D_{0} \subset D_{0}$. Let a mapping $f: D \rightarrow Y$ satisfy the inequality

$$
\left\|2 f\left(\frac{x+y}{2}\right)-f(x)-f(y)\right\| \leq \delta,
$$

for some $\delta \geq 0$ and for all $x, y \in D$. Then there exist a mapping $F: \mathbb{R}^{n} \rightarrow Y$ and a constant $K>0$ such that

$$
2 F\left(\frac{x+y}{2}\right)=F(x)+F(y)
$$

for all $x, y \in \mathbb{R}^{n}$, and

for all $x \in D$.

$$
\|f(x)-F(x)\| \leq K
$$

In section 2 of the present paper, using ideas from the papers of Th. M. Rassias [6] and D. H. Hyers [2], the Hyers-Ulam-Rassias stability of Jensen's equation will be investigated, i.e., the stability of the functional inequality

$$
\left\|2 f\left(\frac{x+y}{2}\right)-f(x)-f(y)\right\| \leq \delta+\theta\left(\|x\|^{p}+\|y\|^{p}\right)
$$

for the case of $p \geq 0(p \neq 1)$ shall be proved. Moreover, by using the same mapping which was constructed by Th. M. Rassias and P. Šemrl [7], we shall show that the inequality (1) is not stable for the case when $p=1$. In section 3 , the HyersUlam stability for Jensen's equation on a restricted domain will be treated, and the result applied to the study of an interesting asymptotic behavior of the additive mappings-more precisely, we prove that a mapping $f: X \rightarrow Y$ satisfying $f(0)=0$ is additive if and only if

$$
\left\|2 f\left(\frac{x+y}{2}\right)-f(x)-f(y)\right\| \rightarrow 0 \quad \text { as } \quad\|x\|+\|y\| \rightarrow \infty .
$$

It should be remarked here that F. Skof [8] has also proved an asymptotic property of the additive mappings. Indeed, she proved that a mapping $f: X \rightarrow Y$ is additive if and only if

$$
\|f(x+y)-f(x)-f(y)\| \rightarrow 0 \quad \text { as } \quad\|x\|+\|y\| \rightarrow \infty .
$$




\section{Hyers-Ulam-Rassias Stability}

First, we prove the Hyers-Ulam-Rassias stability of the Jensen's equation. Assume that $\delta \geq 0$ and $\theta \geq 0$ are fixed.

Theorem 1. Let $p>0$ be given with $p \neq 1$. Suppose a mapping $f: X \rightarrow Y$ satisfies the inequality (1) for all $x, y \in X$. Further, assume $f(0)=0$ and $\delta=0$ in (1) for the case of $p>1$. Then there exists a unique additive mapping $F: X \rightarrow$ $Y$ such that

$$
\|f(x)-F(x)\| \leq \delta+\|f(0)\|+\frac{\theta}{2^{1-p}-1}\|x\|^{p} \quad(\text { for } p<1)
$$

or

$$
\|f(x)-F(x)\| \leq \frac{2^{p-1}}{2^{p-1}-1} \theta\|x\|^{p} \quad(\text { for } p>1),
$$

for all $x \in X$.

Proof. If we put $y=0$ in (1), then we have

$$
\left\|2 f\left(\frac{x}{2}\right)-f(x)\right\| \leq \delta+\|f(0)\|+\theta\|x\|^{p}
$$

for all $x \in X$. By induction on $n$, we prove that

$$
\left\|2^{-n} f\left(2^{n} x\right)-f(x)\right\| \leq(\delta+\|f(0)\|) \sum_{k=1}^{n} 2^{-k}+\theta\|x\|^{p} \sum_{k=1}^{n} 2^{-(1-p) k}
$$

for the case when $0<p<1$. By substituting $2 x$ for $x$ in (6) and dividing by 2 both sides of (6) we see the validity of (7) for $n=1$. Now, assume that the inequality (7) holds true for some $n \in \mathbb{N}$. If we replace $x$ in (6) by $2^{n+1} x$ and divide both sides of (6) by 2 , then it follows from (7) that

$$
\begin{aligned}
& \left\|2^{-(n+1)} f\left(2^{n+1} x\right)-f(x)\right\| \\
& \leq \quad 2^{-n}\left\|2^{-1} f\left(2^{n+1} x\right)-f\left(2^{n} x\right)\right\|+\left\|2^{-n} f\left(2^{n} x\right)-f(x)\right\| \\
& \leq \quad(\delta+\|f(0)\|) \sum_{k=1}^{n+1} 2^{-k}+\theta\|x\|^{p} \sum_{k=1}^{n+1} 2^{-(1-p) k} .
\end{aligned}
$$

This completes the proof of the inequality (7). Let's define

$$
F(x)=\lim _{n \rightarrow \infty} 2^{-n} f\left(2^{n} x\right)
$$

for all $x \in X$. The definition (8) is available because $Y$ is a Banach space and the sequence $\left\{2^{-n} f\left(2^{n} x\right)\right\}$ is a Cauchy sequence for all $x \in X$ : For $n>m$ we use (7) to get

$$
\begin{aligned}
& \left\|2^{-n} f\left(2^{n} x\right)-2^{-m} f\left(2^{m} x\right)\right\| \\
& \quad=2^{-m}\left\|2^{-(n-m)} f\left(2^{n-m} \cdot 2^{m} x\right)-f\left(2^{m} x\right)\right\| \\
& \quad \leq 2^{-m}\left(\delta+\|f(0)\|+\frac{2^{m p}}{2^{1-p}-1} \theta\|x\|^{p}\right) \\
& \quad \rightarrow \quad 0 \text { as } m \rightarrow \infty .
\end{aligned}
$$


Let $x, y \in X$ be arbitrary. It then follows from (8) and (1) that

$$
\begin{aligned}
& \|F(x+y)-F(x)-F(y)\| \\
& \quad=\lim _{n \rightarrow \infty} 2^{-(n+1)}\left\|2 f\left(\frac{2^{n+1}(x+y)}{2}\right)-f\left(2^{n+1} x\right)-f\left(2^{n+1} y\right)\right\| \\
& \leq \lim _{n \rightarrow \infty} 2^{-(n+1)}\left[\delta+\theta 2^{(n+1) p}\left(\|x\|^{p}+\|y\|^{p}\right)\right] \\
& =0 .
\end{aligned}
$$

Hence, $F$ is an additive mapping, and the inequality (7) and the definition (8) imply the validity of (4).

Now, let $G: X \rightarrow Y$ be another additive mapping which satisfies the inequality (4). Then, it follows from (4) that

$$
\begin{aligned}
\|F(x)-G(x)\| & =2^{-n}\left\|F\left(2^{n} x\right)-G\left(2^{n} x\right)\right\| \\
& \leq 2^{-n}\left(\left\|F\left(2^{n} x\right)-f\left(2^{n} x\right)\right\|+\left\|f\left(2^{n} x\right)-G\left(2^{n} x\right)\right\|\right) \\
& \leq 2^{-n}\left(2 \delta+2\|f(0)\|+\frac{2 \theta}{2^{1-p}-1} 2^{n p}\|x\|^{p}\right)
\end{aligned}
$$

for all $x \in X$ and for any $n \in \mathbb{N}$. Since the right-hand side of (9) tends to 0 as $n \rightarrow \infty$, we conclude that $F(x)=G(x)$ for all $x \in X$, which proves the uniqueness of $F$.

For the case when $p>1$ and $\delta=0$ in the functional inequality (1) we can analogously prove the inequality

$$
\left\|2^{n} f\left(2^{-n} x\right)-f(x)\right\| \leq \theta\|x\|^{p} \sum_{k=0}^{n-1} 2^{-(p-1) k}
$$

instead of (7). The rest of the proof for this case goes through in the similar way.

Remark 1. The proof of the Hyers-Ulam-Rassias stability of Jensen's equation for the case of $p=0$ is similar to that of Theorem 1: If a mapping $f: X \rightarrow Y$ satisfies the inequality (1) with $\theta=0$ for all $x, y \in X$, then there exists a unique additive mapping $F: X \rightarrow Y$ satisfying (4) with $\theta=0$.

Remark 2. Let $p \in[0,1)$ be given. By substituting $x+y$ for $x$ and putting $y=0$ in (1) we get

$$
\left\|2 f\left(\frac{x+y}{2}\right)-f(x+y)\right\| \leq \delta+\|f(0)\|+\theta\left(\|x\|^{p}+\|y\|^{p}\right) .
$$

This inequality, together with (1), yields

$$
\|f(x+y)-f(x)-f(y)\| \leq 2 \delta+\|f(0)\|+2 \theta\left(\|x\|^{p}+\|y\|^{p}\right)
$$

for all $x, y \in X$. According to D. H. Hyers [2] and Th. M. Rassias [6] there exists a unique additive mapping $F: X \rightarrow Y$ such that

$$
\|f(x)-F(x)\| \leq 2 \delta+\|f(0)\|+\frac{2 \theta}{1-2^{p-1}}\|x\|^{p}, \quad x \in X,
$$

which is by no means attractive in comparison with (4). 
Remark 3. We also remark that the ideas from the proof of Theorem 1 cannot be applied to the proof of the stability of (1) for the case of $p<0$. An essential process in the proof of Theorem 1 was to put $y=0$ in the inequality (1) which is impossible for the case when $p<0$. The Hyers-Ulam-Rassias stability problem for the case of $p<0$ remains still as an open problem.

Th. M. Rassias and P. Šemrl have constructed in their paper [7] a continuous real-valued mapping to show that the functional inequality

$$
\|f(x+y)-f(x)-f(y)\| \leq \theta(\|x\|+\|y\|)
$$

is not stable in the sense of D. H. Hyers, S. M. Ulam and Th. M. Rassias. By using the result of [7], we prove in the following theorem that the mapping constructed by Rassias and Šemrl serves as a counterexample to Theorem 1 for the case $p=1$.

Theorem 2. The continuous real-valued mapping defined by

$$
f(x)= \begin{cases}x \log _{2}(x+1) & \text { for } x \geq 0, \\ x \log _{2}|x-1| & \text { for } x<0\end{cases}
$$

satisfies the inequality

$$
\left|2 f\left(\frac{x+y}{2}\right)-f(x)-f(y)\right| \leq 2(|x|+|y|),
$$

for all $x, y \in \mathbb{R}$, and the range of $|f(x)-a(x)| /|x|$ for $x \neq 0$ is unbounded for each additive mapping $a: \mathbb{R} \rightarrow \mathbb{R}$.

Proof. It follows from [7] that the mapping $f$ satisfies the inequality

$$
|f(x+y)-f(x)-f(y)| \leq|x|+|y|
$$

for all $x, y \in \mathbb{R}$. By substituting $x / 2$ and $y / 2$ for $x$ and $y$ in (11), respectively, and multiplying both sides by 2 , we have

$$
\left|2 f\left(\frac{x+y}{2}\right)-2 f\left(\frac{x}{2}\right)-2 f\left(\frac{y}{2}\right)\right| \leq|x|+|y|
$$

for any $x, y \in \mathbb{R}$. If we put $x=y$ and divide both sides in (12) by 2 , then we get

$$
\left|f(x)-2 f\left(\frac{x}{2}\right)\right| \leq|x|
$$

for $x \in \mathbb{R}$. By using (12) we obtain

$$
\begin{aligned}
& \left|2 f\left(\frac{x+y}{2}\right)-2 f\left(\frac{x}{2}\right)-2 f\left(\frac{y}{2}\right)\right| \\
& \quad=\left|2 f\left(\frac{x+y}{2}\right)-f(x)-f(y)+f(x)-2 f\left(\frac{x}{2}\right)+f(y)-2 f\left(\frac{y}{2}\right)\right| \\
& \leq|x|+|y|
\end{aligned}
$$

for $x, y \in \mathbb{R}$. The validity of (10) follows immediately from (13) and (14). It is well-known that if an additive mapping $a: \mathbb{R} \rightarrow \mathbb{R}$ is continuous at a point, then $a(x)=c x$, where $c$ is a real number. It is trivial that $|f(x)-c x| /|x| \rightarrow \infty$ as $x \rightarrow \infty$ for any real number $c$, and that the range of $|f(x)-a(x)| /|x|$ for $x \neq 0$ is also unbounded for every non-continuous additive mapping $a: \mathbb{R} \rightarrow \mathbb{R}$, because the graph of the mapping $a$ is everywhere dense in $\mathbb{R}^{2}$. 


\section{Hyers-Ulam Stability on a RESTRICTED domain}

The Hyers-Ulam stability for Jensen's equation on a restricted domain is investigated, and the result is applied to the study of an interesting asymptotic property of additive mappings.

Theorem 3. Let $d>0$ and $\delta \geq 0$ be given. Assume that a mapping $f: X \rightarrow Y$ satisfies the functional inequality

$$
\left\|2 f\left(\frac{x+y}{2}\right)-f(x)-f(y)\right\| \leq \delta
$$

for all $x, y \in X$ with $\|x\|+\|y\| \geq d$. Then there exists a unique additive mapping $F: X \rightarrow Y$ such that

$$
\|f(x)-F(x)\| \leq 5 \delta+\|f(0)\|
$$

for all $x \in X$.

Proof. Suppose $\|x\|+\|y\|<d$. If $x=y=0$, we can choose a $z \in X$ such that $\|z\|=d$. Otherwise, let $z=(1+d /\|x\|) x$ for $\|x\| \geq\|y\|$ or $z=(1+d /\|y\|) y$ for $\|x\|<\|y\|$. It is then obvious that

$$
\begin{aligned}
& \|x-z\|+\|y+z\| \geq d ; \quad\|2 z\|+\|x-z\| \geq d ; \quad\|y\|+\|2 z\| \geq d ; \\
& \|y+z\|+\|z\| \geq d ; \quad\|x\|+\|z\| \geq d .
\end{aligned}
$$

From (15), (17) and the relation

$$
\begin{aligned}
2 f\left(\frac{x+y}{2}\right)-f(x)-f(y)= & 2 f\left(\frac{x+y}{2}\right)-f(x-z)-f(y+z) \\
& -\left[2 f\left(\frac{x+z}{2}\right)-f(2 z)-f(x-z)\right] \\
+ & {\left[2 f\left(\frac{y+2 z}{2}\right)-f(y)-f(2 z)\right] } \\
& -\left[2 f\left(\frac{y+2 z}{2}\right)-f(y+z)-f(z)\right] \\
+ & {\left[2 f\left(\frac{x+z}{2}\right)-f(x)-f(z)\right] }
\end{aligned}
$$

we get

$$
\left\|2 f\left(\frac{x+y}{2}\right)-f(x)-f(y)\right\| \leq 5 \delta .
$$

In view of (15) and (18), the mapping $f$ satisfies the inequality (18) for all $x, y \in X$. Therefore, it follows from (18) and Theorem 1 that there exists a unique additive mapping $F: X \rightarrow Y$ which satisfies the inequality (16) for all $x \in X$.

By using the result of Theorem 3 we now prove an asymptotic behavior of the additive mappings.

Corollary 4. Suppose a mapping $f: X \rightarrow Y$ satisfies the condition $f(0)=0$. Then $f$ is additive if and only if the asymptotic condition (2) holds true. 
Proof. On account of (2), there exists a sequence $\left(\delta_{n}\right)$, monotonically decreasing to 0 , such that

$$
\left\|2 f\left(\frac{x+y}{2}\right)-f(x)-f(y)\right\| \leq \delta_{n}
$$

for all $x, y \in X$ with $\|x\|+\|y\| \geq n$. It then follows from (19) and Theorem 3 that there exists a unique additive mapping $F_{n}: X \rightarrow Y$ such that

$$
\left\|f(x)-F_{n}(x)\right\| \leq 5 \delta_{n}
$$

for all $x \in X$. Let $\ell, m \in \mathbb{N}$ satisfy $m \geq \ell$. Obviously, it follows from (20) that

$$
\left\|f(x)-F_{m}(x)\right\| \leq 5 \delta_{m} \leq 5 \delta_{\ell}
$$

for all $x \in X$, since $\left(\delta_{n}\right)$ is a monotonically decreasing sequence. The uniqueness of $F_{n}$ implies $F_{m}=F_{\ell}$. Hence, by letting $n \rightarrow \infty$ in (20), we conclude that $f$ is additive. The reverse assertion is trivial.

\section{REFERENCES}

[1] G. L. Forti, Hyers-Ulam stability of functional equations in several variables, Aeq. Math. 50 (1995), 143-190. MR 96i:39033

[2] D. H. Hyers, On the stability of the linear functional equation, Proc. Nat. Acad. Sci. U.S.A. 27 (1941), 222-224. MR 2:315a

[3] D. H. Hyers and Th. M. Rassias, Approximate homomorphisms, Aeq. Math. 44 (1992), 125153. MR 93i:39007

[4] Z. Kominek, On a local stability of the Jensen functional equation, Demonstratio Math. 22 (1989), 499-507. MR 91c:39010

[5] J. C. Parnami and H. L. Vasudeva, On Jensen's functional equation, Aeq. Math. 43 (1992), 211-218. MR 93e:39013

[6] Th. M. Rassias, On the stability of the linear mapping in Banach spaces, Proc. Amer. Math. Soc. 72 (1978), 297-300. MR 80d:47094

[7] Th. M. Rassias and P. Šemrl, On the behavior of mappings which do not satisfy Hyers-Ulam stability, Proc. Amer. Math. Soc. 114 (1992), 989-993. MR 92g:47101

[8] F. Skof, Sull'approssimazione delle applicazioni localmente $\delta$-additive, Atti Accad. Sc. Torino 117 (1983), 377-389. MR 89a:39015

[9] S. M. Ulam, Problems in modern mathematics, Chapter VI, Science Editions, Wiley, New York, 1964. MR 43:6031

Mathematics Section, College of Science and Technology, Hong-Ik University, 339800 Cochiwon, South Korea

E-mail address: smjung@wow.hongik.ac.kr 